\title{
METABOLISM OF FERULIC ACID DURING GROWTH OF LACTOBACILLUS PLANTARUM AND LACTOBACILLUS COLLINOIDES
}

\author{
Dries Knockaert ${ }^{1,2}$, Katleen Raes ${ }^{2}$, Christophe Wille ${ }^{2}$, John Van Camp ${ }^{1}$ \\ ${ }^{1}$ : Ghent University, Department of Food Safety and Food Quality, Ghent, Belgium \\ 2: University College West-Flanders, Department of Industrial Engineering and \\ Technology, Kortrijk, Belgium
}

\author{
Dries Knockaert \\ Research Group Food Chemistry and Human Nutrition \\ Department of Food Safety and Food Quality \\ Ghent University \\ Coupure Links 653 \\ 9000 Ghent \\ Belgium \\ e-mail: dries.knockaert@ugent.be \\ Tel: + $32(0) 92646161$
}

Lactic acid fermentation of vegetable products, which are a rich source of phenolic acids, is a technique to prolong the shelf-life. It has been known that phenolic acids, like ferulic acid, may influence the growth of Lactobacillus sp. In addition, different Lactobacillus strains are able to metabolise phenolic compounds leading to metabolites with different structure and bioactivity. This metabolisation may be seen as a tool to improve the bioavailability of micronutrients in these vegetable products. However, more detailed information on the growth kinetics and metabolisation behavior of Lactobacillus sp. as a function of fermentation time is lacking.

In this study, it was aimed to quantify the conversion products of ferulic acid during growth of lactobacillus strains. It was chosen to work with Lactobacillus plantarum (LMG6907) and Lactobacillus collinoides (LMG9194) as lactic acid bacteria. The strains were grown until end stationary phase in MRS medium at $30{ }^{\circ} \mathrm{C}$. Inoculation was done at $1 \%$. The effect of different concentrations of ferulic acid $(0,0.5,1.5$ and $3 \mathrm{mM})$ on growth was tested. Also during growth, samples were taken to identify and quantify ferulic acid and conversion products. Ferulic acid and its conversion products were quantified by LC. Identification of the different conversion products of ferulic acid was done by comparing their absorption spectrum with those of the corresponding standards and by structure profiling using UPLC HDMS-Q-TOF.

From the fermentation experiments, it was observed that none of the ferulic acid concentrations clearly affected the growth of both lactobacilli. However metabolism of ferulic acid by these bacteria was different, and a clear effect of the added ferulic acid concentration was observed. Lactobacillus plantarum converted ferulic acid into 4-vinylguaiacol and hydroferulic acid. The conversion products appeared in different stages on the growth phase, 
and were formed as a function of the added amounts of ferulic acid. Lactobacillus collinoides converted ferulic acid only into 4-vinylguaiacol, however to a much lesser extent compared to Lactobacillus plantarum. From these results, it can be deduced that metabolic pathways on phenolic acids between the different lactic acid bacteria are different. 